\title{
Article
}

\section{Synthesis and Exciton Dynamics of Triplet Sensitized Conjugated Polymers}

Rolf Andernach, Hendrik Utzat, Stoichko Dimitrov, lain

McCulloch, Martin Heeney, James Durrant, and Hugo Bronstein

J. Am. Chem. Soc., Just Accepted Manuscript • DOI: 10.1021/jacs.5b06223 • Publication Date (Web): 22 Jul 2015

Downloaded from http://pubs.acs.org on July 27, 2015

\section{Just Accepted}

"Just Accepted" manuscripts have been peer-reviewed and accepted for publication. They are posted online prior to technical editing, formatting for publication and author proofing. The American Chemical Society provides "Just Accepted" as a free service to the research community to expedite the dissemination of scientific material as soon as possible after acceptance. "Just Accepted" manuscripts appear in full in PDF format accompanied by an HTML abstract. "Just Accepted" manuscripts have been fully peer reviewed, but should not be considered the official version of record. They are accessible to all readers and citable by the Digital Object Identifier (DOI®). "Just Accepted" is an optional service offered to authors. Therefore, the "Just Accepted" Web site may not include all articles that will be published in the journal. After a manuscript is technically edited and formatted, it will be removed from the "Just Accepted" Web site and published as an ASAP article. Note that technical editing may introduce minor changes to the manuscript text and/or graphics which could affect content, and all legal disclaimers and ethical guidelines that apply to the journal pertain. ACS cannot be held responsible for errors or consequences arising from the use of information contained in these "Just Accepted" manuscripts. 


\section{Introduction}

Photoexcited states in organic semiconductors, termed excitons, can have two spin states: a singlet state and a triplet state. In the triplet state, the projections of the respective spin momenta on a given axis are aligned in parallel preventing them from occupying the same space and hence they experience reduced Pauli repulsion. This results in an overall lower energy of the triplet state compared to the singlet. ${ }^{1}$ While the optical transitions between singlet states are typically allowed, the respective transitions between singlet and triplet states are spin-forbidden. Therefore, singlet excitons are normally the primary photoexcitations in organic semiconductors as triplet states cannot be accessed by excitation directly. Their ease of access has allowed singlet excitons to be studied extensively in organic semiconductors. Spectroscopic techniques are routinely employed to measure their properties such as energy levels, lifetime and diffusion length, and thus opened the door for organic semiconductors to be used in optoelectronic devices.

Triplet excitons are, however, of great importance in many optoelectronic devices. In OLEDs, a statistical ratio of 3:1 triplets to singlets is electrically generated via non-geminate hole and electron recombination, hence potentially limiting their efficiency due to the non-emissive nature of the polymer triplets. The incorporation of heavy metal complexes with high phosphorescence quantum yields can allow the utilization of up to $100 \%$ of excitons through the concept of energy harvesting whereby all the excitons are transferred to the metal complex where they can subsequently decay radiatively (phosphorescence) due to the large spin orbit coupling of the heavy metal atom. ${ }^{2-3}$ One drawback, however, shows at high current densities where reductions in efficiency have been observed due to triplet-triplet annihilation. ${ }^{4}$

In polymer:fullerene bulk heterojunction solar cells, population of the polymer triplet state is considered a main loss mechanism. It has been shown, that Charge-Transfer (CT) States, electron-hole pairs bound across the polymer:fullerene interface, can irreversibly recombine to form the polymer triplet excitons due to their lower energy relative to the CT state. ${ }^{5-11}$ Furthermore, the presence of triplet excitons has been linked to the lifetime and stability of conjugated polymers both in neat film and in BHJ devices due to their quenching by oxygen potentially forming highly reactive oxygen species. ${ }^{12}$ Triplet excitons can however be used for the generation of photocurrent when energy levels are appropriately aligned. It has been recently demonstrated for pentacene-based devices utilising singlet fission that $100 \%$ quantum efficiencies can be surpassed. This is possible as singlet fission allows the conversion of one singlet into two triplet excitons each of which is capable of generating charges. ${ }^{13-16}$ In addition, the incorporation of triplet-triplet annihilation (TTA) upconversion layers allow the conversion of IR radiation, normally wasted in solar cells due to their transparence in this wavelength region, into higher energy singlet excitons that are able to generate photocurrent. ${ }^{17-19}$ Despite the ubiquitous presence of triplet excited states in optoelectronic devices 
neither the yield, energetic position nor mobility of polymer triplet excitons are routinely measured, partially due to the challenges of experimentally and theoretically accessing these states. $^{20-21}$

Understanding and controlling the properties of triplet excitons is a key consideration to allow polymers to be used in the next generation of optoelectronics devices. Of particular interest is the determination of polymer triplet exciton mobility. It has been suggested that the longer lifetime of triplet excitons could lead to a longer diffusion length than that of their singlet counterparts. ${ }^{22-26}$ Theoretical predictions have in fact suggested that an enhanced diffusion length could even be achieved with significant disorder present in the system. ${ }^{27}$ Achieving an enhanced exciton diffusion length would allow for the construction of efficient bilayer organic photovoltaic devices, therefore negating the use of the complicated bulk heterojunction. ${ }^{22-23,}{ }^{25-26}$ Conversely, due to the slower Dexter type nature of the triplet energy transfer, triplet excitons have also been suggested to possess lower mobilities than their singlet counterparts. In triplet-triplet annihilation upconversion layers, a lower exciton mobility could be utilized to induce higher local exciton population thus increasing the collision encounter probability.

Experimentally, there have been few reports of triplet exciton diffusion lengths in organic semiconductors. ${ }^{28}$ The hole transport material $N, N^{\prime}$ - $\operatorname{Di}\left(1\right.$-naphthyl)- $N, N^{\prime}$-diphenyl(1,1'-biphenyl)-4,4'-diamine (NPD) has recently been reported to show a triplet diffusion length of $87 \mathrm{~nm},{ }^{29}$ whereas the triplets in structurally related 4,4'-bis(carbazol-9-yl)1,1'biphenyl (CBP) have been reported to have diffusion lengths of 12,25 and $250 \mathrm{~nm}^{30-32} \mathrm{C}_{60}$ was found to possess a triplet diffusion length of $30-35 \mathrm{~nm},{ }^{33}$ and pentacene, which has been extensively studied for its ability to undergo singlet fission, has been shown to have a triplet diffusion length of $40 \mathrm{~nm} .^{34}$ In contrast, structurally related rubrene has been reported to have a triplet diffusion length of $4 \mu \mathrm{m}$ in a single crystal. ${ }^{35}$ For polymeric systems we are aware of only four literature values of triplet exciton mobility. Samiullah et al. reported a triplet diffusion length of almost $4 \mu \mathrm{m}$ in a ladder-type polymer. ${ }^{36}$ Rand et al. reported both the triplet diffusion length of a polyphenylenevinylene poylmer to be $9 \mathrm{~nm}$ (as compared to the $4 \mathrm{~nm}$ single diffusion length). ${ }^{22-23}$ Tamai et al. very recently determined the triplet diffusion lengths of two fluorene based polymers to be 0 and $41 \mathrm{~nm} .{ }^{37}$ It is worth noting how much the triplet properties of two such related structures can differ, highlighting the difficulty as well as the importance of creating the toolset necessary to systematically investigate triplet excited state properties. For polymer based optoelectronics devices to attain their ultimate efficiency and stability, the nature of the triplet exciton must be understood, especially in the next generation of OPV devices and TTA upconversion layers where control over triplet exciton mobility is the key to their success. Consequently, it would be of considerable interest to possess a class of materials where photoexcitation results in a high population of the triplet excitons - hence allowing for their detailed study and potential exploitation.

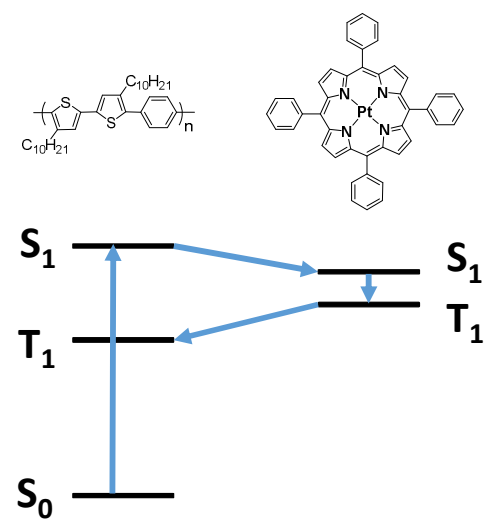

Figure 1: Triplet sensitization of a conjugated polymer using a heavy metal complex

One approach to access the triplet state in the polymer from the photoexcited singlet state is to blend the polymer with heavy metal complexes, which allow the conversion of the singlet to the triplet state due to the strong spin orbit coupling of the heavy metal. For this to occur, careful consideration of the polymer and metal complex energy levels must be taken. The emission of the polymer must overlap with the absorption of the metal complex to allow for efficient energy transfer. Subsequently, the triplet energy level of the polymer must be below that of the complex to allow for back transfer of the triplet excitons (figure 1). This approach has been demonstrated previously, with the aim of producing tripletsensitized solar cells. ${ }^{22,}$ 24, 38-39 However, it has been demonstrated that blends of typically amorphous polymers and highly crystalline metal complexes often lead to extensive phase segregation resulting in a non-homogenous distribution of the metal complex. ${ }^{40-43}$ Furthermore, this aggregation can result in undesirable exciton annihilation processes. ${ }^{43-44}$

To overcome these issues we report here the synthesis and photophysical characterization of a novel polythiophene polymer which includes a Pt-porphyrin complex in the backbone to create a triplet-sensitized conjugated polymer. Our material system allows for the tuning of the incorporation ratio of Pt-porphyrin in the polymer host and for the in-depth study of both the singlet and triplet exciton formation. Incorporation of the metal complex into the polymer backbone prevents phase segregation whilst also simplifying film formation by removing the necessity of blending the two components and may also enhance the energy transfer mechanisms via direct orbital overlap. Furthermore, porphyrins typically show low solubility; incorporation into the polymer backbone circumvents any potential processing issues which can arise as a result of this.

\section{Results and Discussion}

The synthesis of the polymers is shown in Schemes 1 and 2. Mesityldipyrromethane (1) was obtained through a Lewis acid catalyzed condensation reaction of mesitaldehyde in an excess of pyrrole, leading to a white, sheet-like solid. The dipyrromethane was reacted to form the platinated 10,20dibromophenyl-5,15-dimesitylporphyrin (2) in two steps. Reaction of (1) with 4-bromobenzaldehyde proceeded at low concentrations $(\sim 5 \mathrm{mM})$, favoring the ring closure over linear side reactions; aromatization of the porphyrin cores was achieved using the oxidizing agent DDQ. The free base was then reacted with $\mathrm{PtCl}_{2}$, forming the corresponding platinum 
porphyrin complex. The mesityl substituents were chosen so as to impart solubility to the porphyrin and the final polymer, as well as to minimize porphyrin aggregation in the solid state, mediated by the out-of-plane twisting of the meso-subsituents.

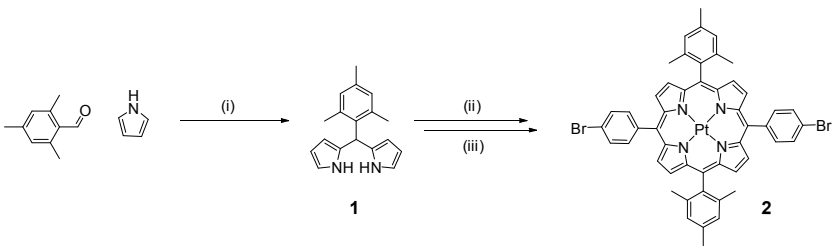

Scheme 1: Synthesis of the porphyrin precursor, (i) $\mathrm{MgBr}_{2}$, TFA, DCM, $50 \%$; (ii) a) DCM, TFA, b) DDQ, $11 \%$; (iii) $\mathrm{PhCN}, \mathrm{PtCl}_{2}$, reflux, $24 \%$

We chose the medium band-gap conjugated backbone poly(phenyl-bithiophene) (TTP) for this study in order to ensure that all the energetic requirements for tripletsensization could occur efficiently. The TTP polymer was synthesized by Suzuki polymerization of 5,5'-dibromo-3,3'didecyl-2,2'-bithiophene with 1,4-benzenediboronic acid bispinacolic ester (Scheme 2). The porphyrin-containing polymers (TTP-Pt) were synthesized via a Suzuki termolecular polymerization, with the different feed ratios and properties given in Table 1. Ter-molecular polymerizations have previously been used to optimize and explore the effects of specific monomers in conjugated polymers.

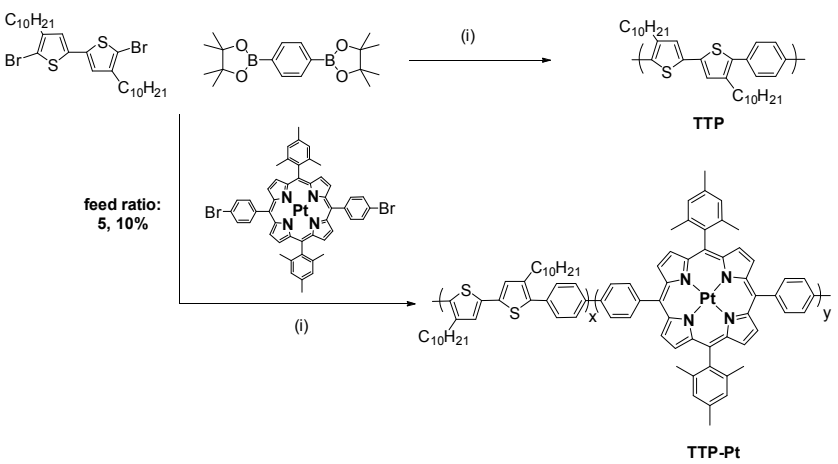

Scheme 2: Synthesis of the TTP and Pt-TTP polymers, (i) $\mathrm{Pd}\left(\mathrm{PPh}_{3}\right)_{4}$, base, toluene, $\operatorname{Ar}, 115^{\circ} \mathrm{C}$

Table 1: Properties of novel polymers

\begin{tabular}{|l|l|l|l|l|l|l|}
\hline & $\begin{array}{l}\mathrm{M}_{\mathrm{n}} \\
{[\mathrm{kDa}]} \\
\mathrm{a})\end{array}$ & $\begin{array}{l}\mathrm{M}_{\mathrm{w}} \\
{[\mathrm{kDa}]} \\
\text { a) }\end{array}$ & PDI & $\begin{array}{l}\mathrm{F}_{\mathrm{R}}{ }^{\mathrm{b}} \\
{[\mathrm{w}-} \\
\%]\end{array}$ & $\begin{array}{l}\mathrm{I}_{\mathrm{R}}{ }^{\mathrm{c}} \\
{[\mathrm{mol}-} \\
\%]\end{array}$ & $\begin{array}{l}\mathrm{I}_{\mathrm{R}}{ }^{\mathrm{c}} \\
{[\mathrm{w}-} \\
\%]\end{array}$ \\
\hline TTP & 44.1 & 73.0 & 1.65 & $0 \%$ & $0 \%$ & $0 \%$ \\
\hline TTP-Pt5 & 10.8 & 19.0 & 1.75 & $5 \%$ & $2 \%$ & $3 \%$ \\
\hline TTP-Pt10 & 14.5 & 24.6 & 1.70 & $\begin{array}{l}10 \\
\%\end{array}$ & $5 \%$ & $8 \%$ \\
\hline
\end{tabular}

a) Determined by GPC using a polystyrene standard.; b) Feed ratio $\left(\mathrm{F}_{\mathrm{R}}\right)$ determined by weight per repeat unit.; c) Incorporation ratios $\left(I_{R}\right)$ determined by integration of the ${ }^{1} \mathrm{H}$ NMR porphyrin signal per repeat unit (referenced to the integral of the thiophene aromatic signal).

TPP was isolated as a bright yellow solid with high visible fluorescence, whereas all the porphyrin containing polymers were visibly less fluorescent. Reasonable molecular weights were obtained for all the resulting polymers, with a trend for decreasing molecular weight at higher porphyrin dopant ratios. We believe this to be due to a slight loss in solubility for the resulting polymers which resulted in a quicker precipitation of the polymer chains during the synthesis. We note the lower molecular weight of the porphyrin containing polymers may affect their photophysical properties somewhat, but we believe that due to the low molecular weight of the repeat units the degree of polymerization is sufficiently high for them to be considered polymeric. The incorporation of the platinum porphyrin was verified by ${ }^{1} \mathrm{H}$ NMR spectroscopy (Supporting Information S2), the aromatic signals of the thiophene (7.70 ppm) and phenyl groups (7.51 ppm) clearly attributed and their integrals matching. The porphyrin peaks $(8.9-8.0 \mathrm{ppm})$ of the $10 \mathrm{w}-\%$ doped polymer can be seen in comparison, with each peak integral corresponding to four protons on the porphyrin substructure. In the polymers containing higher weight percentages of porphyrin a secondary smaller set of porphyrin peaks can be observed. This is likely to be due to a terminal porphyrin on the polymer chain as opposed to the above species which are incorporated into the conjugated polymer on both sides. Overall, the incorporation ratio of the porphyrin was slightly lower than the feed ratio for all concentrations which possibly stems from a lower reactivity of the porphyrin monomer relative to the bithiophene species.

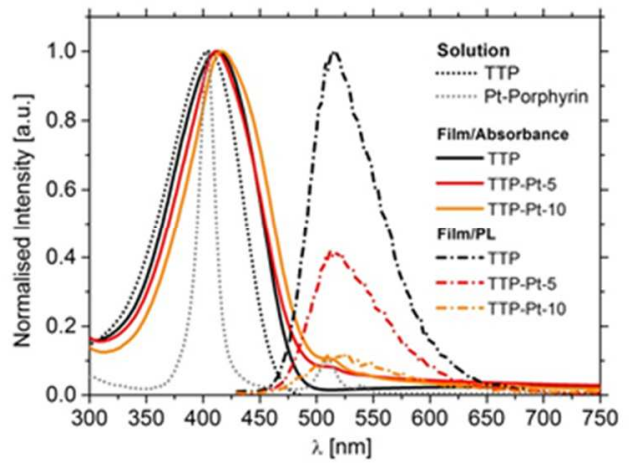

Figure 2: Absorption and Emission spectra of novel polymers

Figure 2 displays the UV-VIS spectra of TTP, TTP-PT-5 and TTP-Pt-10 polymers in solution and thin films spin-coated on glass. All thin film spectra are weakly red-shifted compared to the solution, likely because of solid state packing effects commonly observed in conjugate polymers (404 nm compared to $419 \mathrm{~nm}$ for TTP). The absorption maximum of the TTP polymer and the TTP-Pt5 and TTP-Pt10 polymers is around $\lambda \max =416 \mathrm{~nm}$ and have similar onset of polymer absorption. These findings indicate that the addition of the Pt-porphyrin complex and the difference in molecular weight, as determined by GPC, has no noticeable effect on the effective conjugation length of the polymer. The Pt-porphyrin complex exhibits two distinct absorption features around $405 \mathrm{~nm}$ and at $510 \mathrm{~nm}$. The polymers show emission around $525 \mathrm{~nm}$ which we assign to the fluorescence of the TTP backbone. Both, the absorption and emission energies are consistent with previously reported values for TTP. ${ }^{46-47}$ With increasing content of Pt-porhyrin in the backbone, the fluorescence intensity decreases (Figure 2), indicating an efficient singlet exciton quenching, which we tentatively assign to quenching induced by the Pt-porphyrin backbone. 

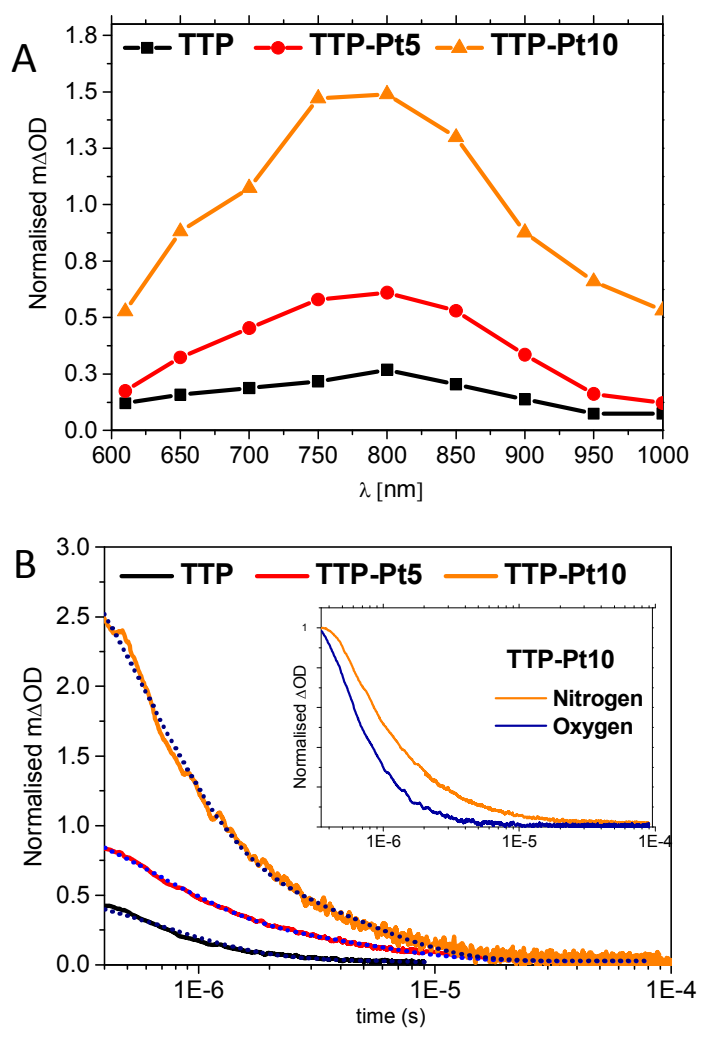

Figure 3: Transient absorption spectra of TTP, TTP-Pt-5 and TTP-Pt-10 measured at $800 \mathrm{~ns}$ after photoexcitation $(420 \mathrm{~nm})$ (A) and the corresponding decay dynamics measured at $800 \mathrm{~nm}$ (B). The insert in (B) shows the effect of moving from a nitrogen atmosphere (used for all experiments unless otherwise stated) to oxygen for TTP-Pt-10. Analogous acceleration of decay dynamics in the presence of oxygen were observed for all three polymers.

In order to study the singlet and triplet exciton dynamics and the mechanism of the triplet exciton formation in TTP, TTP-Pt-5 and TTP-Pt-10, we used Transient Absorption Spectroscopy (TAS) on multiple timescales. First, we performed microsecond TAS of the polymer thin films spincoated on glass to determine the triplet exciton yields and triplet exciton decay dynamics in the three polymers studied. Figure 3 (A) presents the TAS spectra in the visible spectral region at $800 \mathrm{~ns}$ after photoexcitation of the TTP backbone $(420 \mathrm{~nm})$ for TTP, TTP-Pt5 and TTP-Pt10. The corresponding decays of the transient absorption are presented in figure 3 (B). For TTP, TTP-Pt-5 and TTP-Pt-10 a similarly broad transient absorption signal at around $800 \mathrm{~nm}$ is observed. This signal is strongly quenched by the presence of oxygen for all samples, as it is shown for TTP-Pt-10 in the inset of figure 3 (B). The decay of the transient absorption of the pristine TTP polymer was fitted well with a mono-exponential function with $\tau_{\text {TTP }}=501 \pm 3 \mathrm{~ns}$. Therefore, based on the similarity of the spectra and the observed oxygen quenching, we assign the signal to the transient absorption of the triplet exciton on the TTP backbone. Previously, Ohkita et al. reported the transient absorption maximum of the TTP polymer triplet exciton around $700 \mathrm{~nm}$ which is in close agreement with our observed value. $^{46}$
A comparison of the transient absorption between the three polymers reveals a clear increase of the initial signal amplitude with the incorporation ratio of Pt-porphyrin, which shows an improved triplet generation yield in the presence of the metal complex in the polymer backbone. In addition, a change in the decay dynamics of the triplet exciton of TTP-Pt5 and TTP-Pt-10 compared to the non porphyrin containing TTP polymer is observed. While the decay of the triplet exciton of TTP follows a mono-exponential dynamics, the relaxation of the triplet exciton in the TTP-Pt polymers can only be fitted with bi-exponential functions yielding $\tau_{1}$-TTP$\mathrm{Pt} 5=440 \pm 3 \mathrm{~ns}$ and $\tau_{2}$-TTP-Pt5 $=3.55 \pm 0.03 \mu \mathrm{s} ; \tau_{1}$-TTPPt10 $=380 \pm 3$ ns and $\tau_{2}$-TTP-Pt10 $=3.16 \pm 0.03 \mu \mathrm{s}$. The $\mu \mathrm{s}$ decay component is not associated with any spectral evolution. In the course of our discussion we will provide a possible explanation for the altered exciton decay dynamics of the TTP-Pt polymers compared to the TTP polymer.

In order to elucidate the singlet exciton dynamics and the triplet exciton formation we utilized ultrafast TAS to measure thin films of TTP, TTP-Pt-5 and TTP-Pt-10. The spectra of TTP and TTP-Pt-10 up to 5.8 ns are presented in Figure 4.
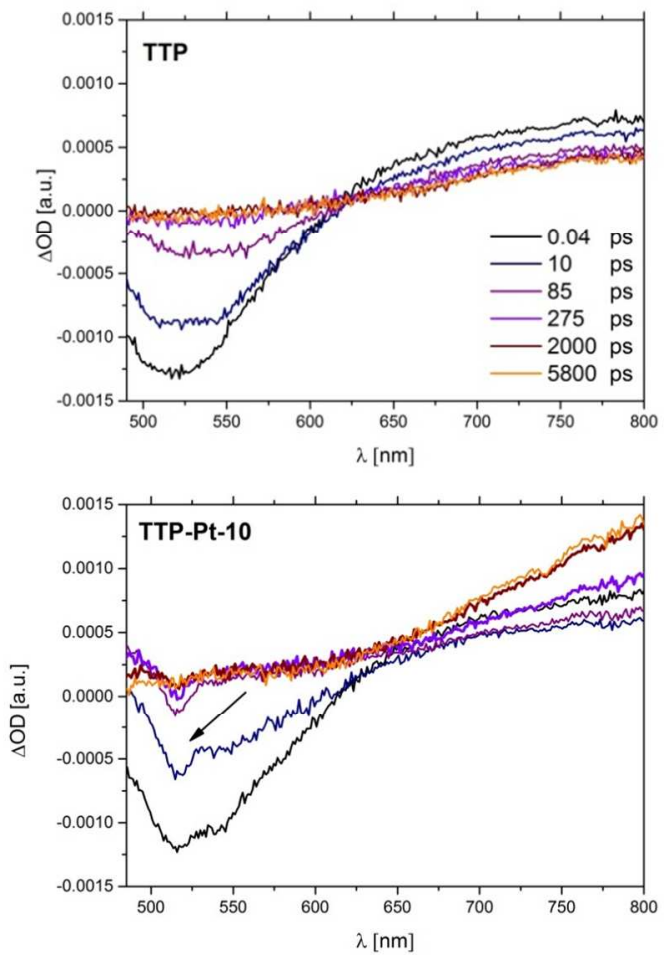

Figure 4: TAS spectra of TTP and TTP-Pt-10 films at delay times of $0.04-5800 \mathrm{ps}$ after photoexcitation of the TTP $\left(\lambda_{\text {exc }}: 420 \mathrm{~nm}\right)$.

The spectra of the neat TTP polymers exhibit three characteristic features. First, a negative, quickly relaxing transient absorption at around $525 \mathrm{~nm}$ is observed, disappearing on the $10-100$ ps timescale. Comparison to the steady state PL spectra (see figure 2) allows us to assign this feature to stimulated emission from the TTP singlet exciton. Second, a strong, fast decaying transient absorption in the red spectral region is observed. This transient absorption signal can be assigned to absorption of the singlet exciton of the TTP backbone which exhibits an absorption maximum in the near IR spectral region (see SI 3). Following the rapid decay of these two signals, assigned to singlet exciton decay, a residual broad transient absorption with increasing amplitude from 500 
$\mathrm{nm}$ to $800 \mathrm{~nm}$ is observed, which does not evolve over the timescale measured (up to $5.8 \mathrm{~ns}$ after photoexcitation). This broad, long lived transient absorption matches the spectral shape of the triplet exciton absorption measured at $800 \mathrm{~ns}$ delay (figure 3 (A)) after photoexcitation and can thus be assigned to the triplet exciton on the TTP backbone.

The spectra of the TTP-Pt-10 polymer exhibit similar features as the TTP polymer, but an additional negative, relatively narrow transient absorption feature is observed at around $510 \mathrm{~nm}$, most prominently in the spectra at time delays of 85 and 275 ps, as shown in figure 4 (arrow). We assign this feature to the ground state bleach (GSB) of the Pt-porphyrin (compare to GS spectrum in figure 2). We note that ISC from singlet to triplet excited states in the Pt-containing organic molecules and other heavy metal porphyrins have been observed to be very fast $(<200 \mathrm{fs}-1 \mathrm{ps})$, significantly faster than the lifetime of this feature. We thus assign this Pt-porphyrin GSB signal specifically to the presence of Pt-porphyrin triplet states. $^{48-50}$

Based on the assignments made, we were able to extract the excited state population dynamics from the transient absorption spectra. Specifically the amplitude of the broad TTP stimulated emission band at $525 \mathrm{~nm}$ was used as an assay of TTP singlet excitons. The triplet TTP absorption at $800 \mathrm{~nm}$ was determined by subtracting the polymer singlet absorption from the total photoinduced absorption at this wavelength, assuming a negligible triplet yield early (200 fs) after photoexcitation. The triplet photoinduced absorption was used to estimate the TTP triplet exciton population rise in the films. Finally, the amplitude of the narrow GSB feature of the Ptporphyrin complex at $510 \mathrm{~nm}$ was used as a measure of the triplet state population of this complex. The resulting transient decays are presented in figure 5.

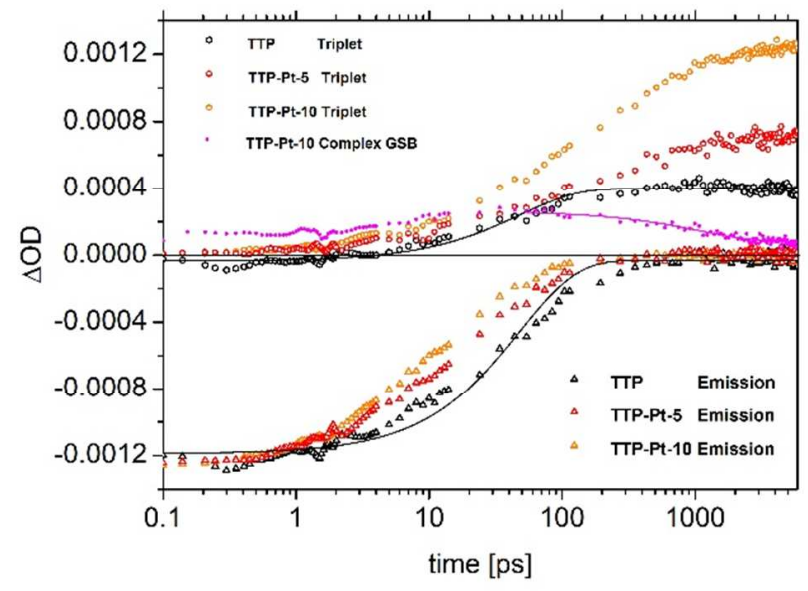

Figure 5: Dynamics of spectral features observed from transient absorption spectra such as that shown in figure 4, comprising the $525 \mathrm{~nm}$ stimulated emission assigned to TTP singlet excitons (triangles), the photoinduced absorption assigned to TTP triplet excitons (circles) and $510 \mathrm{~nm}$ GSB signal assigned to Ptporphyrin triplet states (dotted line). Lines represent least square fits of these population dynamics using monoexponential functions.

We focus first on the TTP polymer (figure 5, black traces). As the stimulated emission of the singlet exciton relaxes (black triangles), the triplet exciton absorption rises (black circles). Both, the stimulated emission decay and the TTP triplet rise were fitted with monoexponential functions with a shared time constant of $48.0 \pm 1.4$ ps, indicating direct population conversion from the singlet to the triplet exciton manifold. This time constant relates to the singlet exciton lifetime in the TTP polymer, in which the triplet excitons are formed via intersystem crossing. Similar lifetimes have previously been reported for other medium bandgap polythiophene polymers such as $\mathrm{P}_{3} \mathrm{HT}^{51}$ By taking into account the extinction coefficient of the TTP triplet exciton $\varepsilon$ $(800 \mathrm{~nm})=80.000 \mathrm{M}^{-1} \mathrm{~cm}^{-1}$ (see SI4 for calculation of $\varepsilon$ ), we estimated the efficiency of the ISC in the neat polymer to be $20 \%$, calculated from the ratio of the yields of the photoexcited singlet excitons and the generated triplet excitons. Given that

$$
\text { ISC }_{\text {efficiency }}=\frac{\boldsymbol{k}_{I S C}}{k_{0}}
$$

and using our estimates for the singlet exciton decay rate constant $k_{0}$ of $(48 \mathrm{ps})^{-1}=2.1 \times 10^{10} \mathrm{~s}^{-1}$ and $I S C_{\text {eff }}=20 \%$, we obtain $\mathrm{k}_{\mathrm{ISC}}=(200 \pm 10 \mathrm{ps})^{-1}=4.2 \times 10^{9} \mathrm{~s}^{-1}$. The relatively high ISC efficiency in the polymer can be attributed to the incorporation of a higher homologue atom, (i.e. sulfur, in the polythiophene polymer exhibiting a high spin-orbit coupling constant) or residual palladium from the polymerization. ${ }^{52}$

The stimulated emission decay in TTP-Pt-10 (figure 5, orange traces) occurs on a faster timescale compared to the neat TTP, as expected from the observed TTP fluorescence quenching in this sample. It is striking that for this film, unlike for the TTP film, the rise of TTP triplet absorption is significantly delayed relative to the decay of this stimulated emission. The decay of the TTP stimulated emission approximately coincides with the appearance of the Ptporphyrin GSB, whilst the rise of the TTP triplet absorption coincides with the decay of this GSB. This is strongly indicative of efficient TTP singlet exciton quenching by the Pt-porphyrin complex on the 10's ps timescale and a subsequent slower back transfer of the complex's triplet state to the polymer (figure 6).

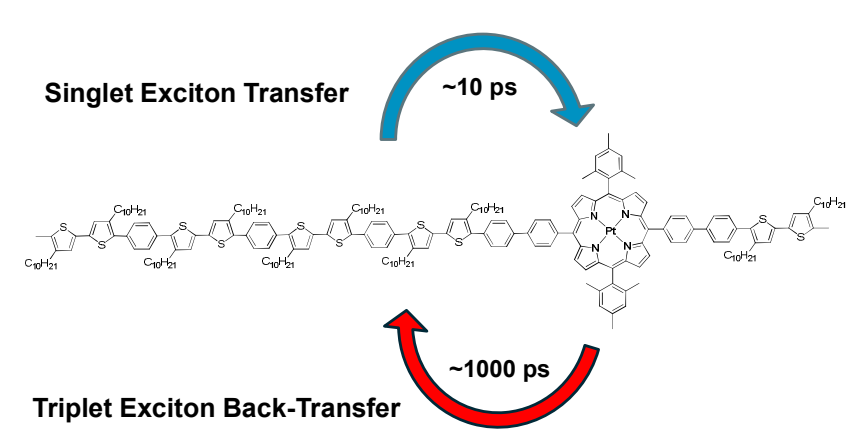

Figure 6: Diagram showing triplet sensitization process

Pt-Porphyrins such as the one studied here are known to undergo very fast and efficient ISC, thus here we assume that after initial population of the Pt-porphyrin singlet, the porphyrin undergoes extremely fast and efficient ISC, previously estimated to be $<200 \mathrm{fs}^{49-50} \mathrm{We}$ also assume a homogenous distribution of Pt-porphyrin quencher sites in the film because the covalent incorporation of the Pt-porphyrin as a quencher molecule mitigates phase segregation between the materials. Therefore, we used a single exponential function to fit the decay of the Pt-porphyrin GSB, which we associated with the triplet back transfer to the TTP triplet, giving a time 
constant of $1087 \pm 104$ ps. Due to spin conservation rules this triplet energy transfer can only occur via short-range Dexter electron exchange mechanism, which depends strongly on the orbital offset as well as on the orbital overlap between the electron exchanging entities. According to our results, this process is therefore the rate limiting step in the overall process of triplet exciton formation in the TTP-Pt polymers. The time constant for the overall process of TTP triplet formation is of a similar magnitude to the previously reported time constant for mediated triplet exciton formation in polyfluorene-Iridium complex blends: $0.28 \mathrm{~ns} .^{53}$ In addition to the rate constant of triplet population, we also estimated the efficiency of the triplet exciton back transfer by simply taking the ratio of the GSB of the Pt-porphyrin at $5.5 \mathrm{~ns}$ and $30 \mathrm{ps}$, revealing very high triplet exciton generation efficiency of $\sim 60 \%$.

One of the properties of triplet excitons in organic semiconductors most relevant to optoelectronic applications is their diffusion length. Here, we carried out transient absorption spectroscopy on the $\mu$ s timescale as a function of excitation density to study the decay dynamics of the triplets in the TTP polymer and TTP-Pt-10 (Figure 7). Triplet-triplet exciton annihilation is a well-documented process taking place at increased excitation densities when the encounter of two triplets can lead to the generation of one singlet exciton. This process is expectedly strongly dependent on the triplet exciton diffusion constant of the material studied and as such can be used for estimation of triplet exciton diffusion lengths.

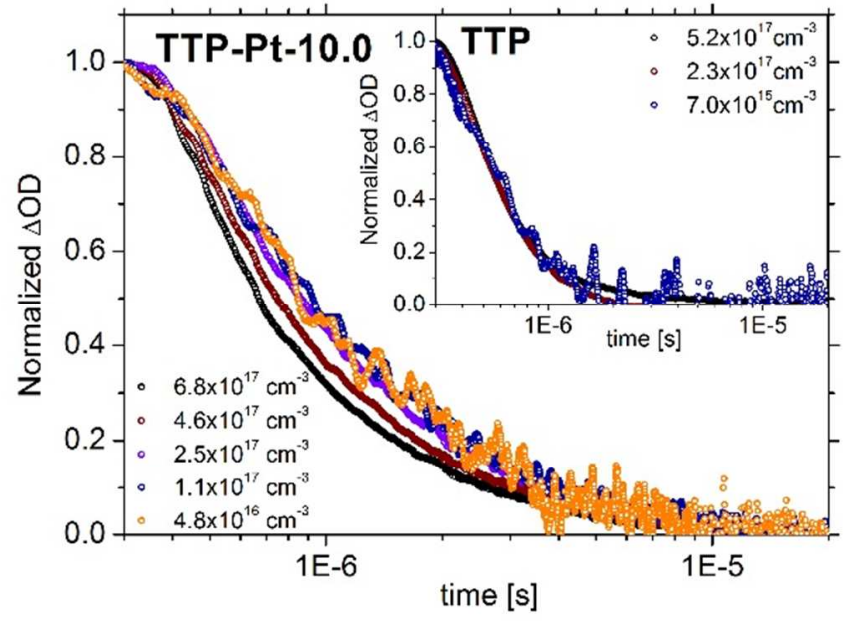

Figure 7: Decay dynamics of the triplet excitons at different initial triplet densities for TTP-Pt-10. The inset shows the corrresponding kinetics of the neat TTP polymer. The densities were calculated on the basis of the extinction coefficient of the TTP triplet exciton and the film thickness.

The TTP polymer showed triplet exciton decay completely independent from the initial triplet yield, at least in the range of triplet exciton densities studied here (figure 7 , inset). On the basis of our triplet density dependent decay dynamics, we were able to estimate an upper limit for the triplet exciton diffusion in the TTP. The highest triplet density created upon laser excitation in the TTP polymer corresponds to an initial mean separation distance between triplets of $12.4 \mathrm{~nm}$. The absence of any TTA indicates that the triplet exciton diffusion length in the pristine TTP polymer is below this value.

For the TTP-Pt-10 polymer we observed that the decay dynamics of the triplet excitons is independent of the initial triplet exciton concentration up until an exciton density of 2.5 x $10^{17} \mathrm{~cm}^{-3}$ is reached. However, upon increasing the initial triplet density to $4.6 \times 10^{17} \mathrm{~cm}^{-3}$ via using higher light excitation density to pump the sample an accelerated population decay is observed. Notably, the TTA in TTP-Pt-10 occurs at triplet densities which do not cause annihilation processes in the TTP polymer. The triplet excitons in TTP-Pt10 are formed predominantly due to the energy transfer from the Pt-porphyrin complex and are thus generated in higher densities around the Pt-porphyrin centers. In turn, this inhomogeneous distribution of excitons may result in a higher probability of TTA in TTP-Pt-10 compared to the TTP polymer, where in the latter the triplets are formed via ISC homogeneously. Based on these observations, we conclude that the polymer triplet exciton is confined in close proximity to the Pt-porphyrin complex, perhaps due to a low triplet exciton mobility. As noted earlier we observe the triplet exciton decay for TTP-Pt polymers is biphasic (Figure $3 \mathrm{~b}$ ) and its lifetime is significantly increased $(>3 \mu \mathrm{s})$ whereas the triplet lifetime of the neat TTP polymer is $\sim 500$ ns only (as determined by monoexponential fitting of its decay (Figure $3 b)$ ). The excited state lifetime of Pt-porphyrin complexes is typically $>40 \mu \mathrm{s} .{ }^{54}$ This suggests that the triplets generated via the platinum complex are in equilibrium with the triplet state of the complex (Figure 8). The proposition that the Ptporphyrin and TTP triplet states are in equilibrium is consistent with the observed incomplete triplet back transfer $(60 \%)$ from the Pt-porphyrin to the TTP backbone. A similar triplet state equilibria has been reported for conjugated small molecules but has never been demonstrated in a conjugated polymer. ${ }^{55-56}$ Using a Boltzmann distribution, we can therefore estimate the triplet free energy level of the polymer to be $\sim 0.06 \mathrm{eV}$ below that of the Pt-complex. The phosphorescence emission maxima of Pt -TPP is $1.86 \mathrm{eV}$ and thus we can estimate the polymer triplet energy level to be $\sim 1.93 \mathrm{eV}$. The $\mathrm{S}-\mathrm{T}$ gap can subsequently be estimated to be $0.65 \mathrm{eV}$ which is in accordance with studies suggesting that the exchange energy in conjugated polymers is approximately $0.7 \mathrm{eV} .^{57-58}$ Such drastic manipulation of local triplet exciton density and lifetime which result in increased T-T annihilation events and has significant implications for triplet-triplet annihilation upconversion layers. It is proposed that utilizing low triplet exciton mobility materials may lead to non-diffusion controlled TTA-UC materials whereby high local triplet density is used to increase upconversion annihilation efficiency. 


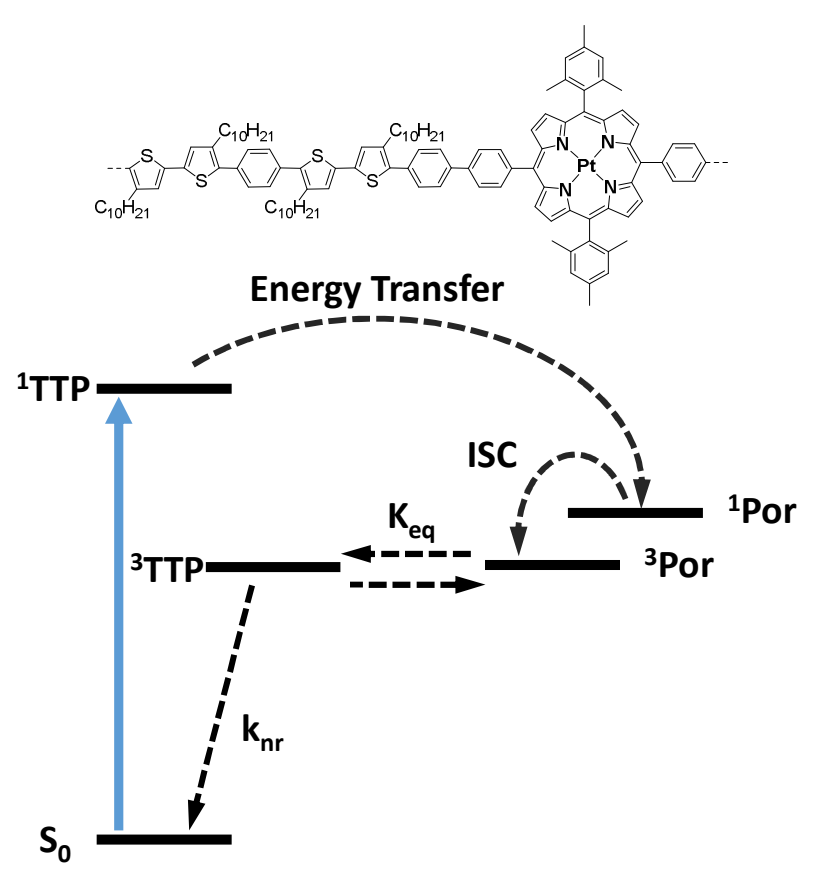

Figure 8: Proposed exciton transfer and equilibration processes in TTP-Pt polymer films.

\section{Conclusion}

We report the synthesis of a series of novel polythiophenebased semiconducting polymers suitable for efficient polymer triplet exciton generation. The triplet formation is achieved by conversion of the singlet excitons using a host-guest-approach which covalently incorporates heavy metal platinum porphyrin into the conjugated backbone. This approach of incorporating the complex into the polymer backbone allows for an efficient generation of triplet excitons in the polymer material while avoiding potential phase segregation between the polymer backbone and the Pt-porphyrin complex in thin films.

We obtained a detailed photophysical picture of the singlet and triplet exciton dynamics and the triplet exciton formation mechanism using transient absorption spectroscopy. Initial singlet photoexcitations and transferred to the Pt-porphyrin which undergoes ISC. The triplets of the complex are subsequently transferred back onto the polymer triplet energy level with an efficiency of $60 \%$ and a time constant of $1087 \pm 104$ ps, indicating a slow, Dexter electron exchange process as the underlying mechanism. By measuring the triplet density dependent triplet decay dynamics in the TTP polymer we were able to define an upper limit for the triplet exciton diffusion length of $12.4 \mathrm{~nm}$. We associate the incomplete triplet backtransfer to an equilibrium between the triplet states of the polymer and the complex which is causing a prolonged polymer triplet exciton lifetime in platinum containing polymers. This results in an increase in local triplet exciton density and increased triplet-triplet annihilation events potentially allowing for a new method of producing upconversion layers.

Our findings show that the sensitization of semiconducting polymers with triplet excitons can be very efficient. This novel materials system allows for determination of almost all the photophysical parameters of a conjugated polymer including estimations for the polymer triplet energy level and triplet diffusion lengths using only spectroscopic methods on thin films. Finally, we demonstrate that this novel host-guest polymeric system can be used to manipulate the spatial distribution of triplet excitons and their lifetime which we believe has significant implications for both triplet sensitized OPV devices and triplet-triplet annihilation up-conversion layers.

\section{ASSOCIATED CONTENT}

Supporting Information. Synthetic details and photophysical characterization are shown. http://pubs.acs.org."

\section{AUTHOR INFORMATION}

\section{Corresponding Author}

*h.bronstein@ucl.ac.uk,j.durrant@imperial.ac.uk

\section{Author Contributions}

$\dagger$ These authors contributed equally.

\section{ACKNOWLEDGEMENTS}

We are grateful to the Imperial College JRF scheme for funding. We are also grateful to the EPSRC, EP/I019278/1 for funding.

\section{REFERENCES}

(1) Köhler, A.; Bässler, H., Mater. Sci. Eng. R-Rep. 2009, 66, 71.

(2) Adachi, C.; Baldo, M. A.; Thompson, M. E.; Forrest, S. R., J. Appl. Phys. 2001, 90, 5048.

(3) Baldo, M. A.; O’Brien, D. F.; Thompson, M. E.; Forrest, S. R., Phys. Rev. B 1999, 60, 14422 .

(4) Reineke, S.; Walzer, K.; Leo, K., Phys. Rev. B 2007, 75, 125328 .

(5) Rao, A.; Chow, P. C. Y.; Gelinas, S.; Schlenker, C. W.; Li, C.Z.; Yip, H.-L.; Jen, A. K. Y.; Ginger, D. S.; Friend, R. H., Nature 2013, 500, 435.

(6) Chow, P. C. Y.; Gélinas, S.; Rao, A.; Friend, R. H., J. Am. Chem. Soc. 2014, 136, 3424.

(7) Westenhoff, S.; Howard, I. A.; Hodgkiss, J. M.; Kirov, K. R.; Bronstein, H. A.; Williams, C. K.; Greenham, N. C.; Friend, R. H., J. Am. Chem. Soc. 2008, 130, 13653.

(8) Clarke, T. M.; Durrant, J. R., Chem. Rev. 2010, 110, 6736.

(9) Schlenker, C. W.; Chen, K.-S.; Yip, H.-L.; Li, C.-Z.; Bradshaw, L. R.; Ochsenbein, S. T.; Ding, F.; Li, X. S.; Gamelin, D. R.; Jen, A. K. Y.; Ginger, D. S., J. Am. Chem. Soc. 2012, 134, 19661.

(10) Liedtke, M.; Sperlich, A.; Kraus, H.; Baumann, A.; Deibel, C.; Wirix, M. J. M.; Loos, J.; Cardona, C. M.; Dyakonov, V., J. Am. Chem. Soc. 2011, 133, 9088.

(11) Dimitrov, S. D.; Wheeler, S.; Niedzialek, D.; Schroeder, B. C.; Utzat, H.; Frost, J. M.; Yao, J.; Gillett, A.; Tuladhar, P. S.; McCulloch, I.; Nelson, J.; Durrant, J. R., Nat Commun 2015, 6:6501

(12) Soon, Y. W.; Cho, H.; Low, J.; Bronstein, H.; McCulloch, I.; Durrant, J. R., Chem. Commun. 2013, 49, 1291.

(13) Congreve, D. N.; Lee, J.; Thompson, N. J.; Hontz, E.; Yost, S. R.; Reusswig, P. D.; Bahlke, M. E.; Reineke, S.; Van Voorhis, T.; Baldo, M. A., Science 2013, 340, 334.

(14) Smith, M. B.; Michl, J., Annu. Rev. Phys. Chem. 2013, 64, 361.

(15) Rao, A.; Wilson, M. W. B.; Hodgkiss, J. M.; Albert-Seifried, S.; Bässler, H.; Friend, R. H., J. Am. Chem. Soc. 2010, 132, 12698.

(16) Musser, A. J.; Liebel, M.; Schnedermann. C.; Wende, T.; Kehoe, T. B.; Rao, A.; Kukura, P., Nature Physics 2015, 11, 352

(17) Zhao, J.; Ji, S.; Guo, H., RSC ADV 2011, 1, 937.

(18) Singh-Rachford, T. N.; Castellano, F. N., Coord. Chem. Rev. 2010, $254,2560$.

(19) Cui, X.; Zhao, J.; Zhou, Y.; Ma, J.; Zhao, Y., J. Am. Chem. Soc. 2014, 136, 9256.

(20) Reineke, S.; Baldo, M. A., Sci. Rep. 2014, 4, 3797.

(21) Kohler, A.; Bassler, H., J. Mater. Chem. 2011, 21, 4003. 
(22) Rand, B. P.; Schols, S.; Cheyns, D.; Gommans, H.; Girotto, C.; Genoe, J.; Heremans, P.; Poortmans, J., Org. Electron. 2009, 10, 1015.

(23) Rand, B. P.; Girotto, C.; Mityashin, A.; Hadipour, A.; Genoe, J.; Heremans, P., Appl. Phys. Lett. 2009, 95, 173304

(24) Lee, C.-L.; Hwang, I.-W.; Byeon, C. C.; Kim, B. H.; Greenham, N. C., Adv. Funct. Mater. 2010, 20, 2945.

(25) Winroth, G.; Podobinski, D.; Cacialli, F., J. Appl. Phys. 2011 110

(26) Xiong, K.; Hou, L.; Wang, P.; Xia, Y.; Chen, D.; Xiao, B., J. Lumin. 2014, 151, 193.

(27) Yost, S. R.; Hontz, E.; Yeganeh, S.; Van Voorhis, T., J. Phys. Chem. C 2012, 116, 17369.

(28) Mikhnenko, O. V.; Blom, P. W. M.; Nguyen, T.-Q., Energy Environ. Sci. 2015, 8, 1867

(29) Mikhnenko, O. V.; Ruiter, R.; Blom, P. W. M.; Loi, M. A., Phys. Rev. Lett. 2012, 108, 137401.

(30) Lebental, M.; Choukri, H.; Chénais, S.; Forget, S.; Siove, A.; Geffroy, B.; Tutiš, E., Phys. Rev. B 2009, 79, 165318.

(31) Giebink, N. C.; Sun, Y.; Forrest, S. R., Org. Electron. 2006, 7, 375 .

(32) Matsusue, N.; Ikame, S.; Suzuki, Y.; Naito, H., J. Appl. Phys. 2005, 97, 123512

(33) Qin, D.; Gu, P.; Dhar, R. S.; Razavipour, S. G.; Ban, D., Physica Status Solidi A 2011, $208,1967$.

(34) Tabachnyk, M.; Ehrler, B.; Bayliss, S.; Friend, R. H.; Greenham, N. C., Appl. Phys. Lett. 2013, 103, 153302

(35) Irkhin, P.; Biaggio, I., Phys. Rev. Lett. 2011, 107, 017402.

(36) Samiullah, M.; Moghe, D.; Scherf, U.; Guha, S., Phys. Rev. B 2010, 82, 205211.

(37) Tamai, Y.; Ohkita, H.; Benten, H.; Ito, S., Chem. Mater. 2014, 26,2733 .

(38) Luhman, W. A.; Holmes, R. J., Appl. Phys. Lett. 2009, 94, 153304

(39) Roberts, S. T.; Schlenker, C. W.; Barlier, V.; McAnally, R. E.; Zhang, Y.; Mastron, J. N.; Thompson, M. E.; Bradforth, S. E., J. Phys. Chem. Lett. 2010, 2, 48.

(40) Chen, F.-C.; He, G.; Yang, Y., Appl. Phys. Lett. 2003, 82, 1006.
(41) Chen, F.-C.; Chang, S.-C.; He, G.; Pyo, S.; Yang, Y.; Kurotaki, M.; Kido, J., J. Polym. Sci., Part B: Polym. Phys. 2003, 41 , 2681.

(42) Noh, Y.-Y.; Lee, C.-L.; Kim, J.-J.; Yase, K., J. Chem. Phys. 2003, 118, 2853

(43) Jankus, V.; Snedden, E. W.; Bright, D. W.; Whittle, V. L.; Williams, J. A. G.; Monkman, A., Adv. Funct. Mater. 2013, 23, 384.

(44) Kraft, A.; Grimsdale, A. C.; Holmes, A. B., Angew. Chem. Int. Ed. 1998, 37, 402 .

(45) Fang, L.; Zhou, Y.; Yao, Y.; Diao, Y.; Lee, W.; Appleton, A. L.; Allen, R.; Reinspach, J.; Mannsfeld, S. C. B.; Bao, Z., Chem. Mater. 2013, 25, 4874

(46) Ohkita, H.; Cook, S.; Astuti, Y.; Duffy, W.; Tierney, S.; Zhang, W.; Heeney, M.; McCulloch, I.; Nelson, J.; Bradley, D. D. C.; Durrant, J. R., J. Am. Chem. Soc. 2008, 130, 3030.

(47) Ohkita, H.; Cook, S.; Astuti, Y.; Duffy, W.; Heeney, M.; Tierney, S.; McCulloch, I.; Bradley Donal, D. C.; Durrant James, R., Chem. Commun. 2006, 3939.

(48) Forster, L. S., Coord. Chem. Rev. 2006, 250, 2023.

(49) Juban, E. A.; Smeigh, A. L.; Monat, J. E.; McCusker, J. K., Coord. Chem. Rev. 2006, 250, 1783.

(50) Sheng, C. X.; Singh, S.; Gambetta, A.; Drori, T.; Tong, M.; Tretiak, S.; Vardeny, Z. V., Sci. Rep. 2013, 3, 2653.

(51) Tamai, Y.; Matsuura, Y.; Ohkita, H.; Benten, H.; Ito, S., The Journal of Physical Chemistry Letters 2014, 5, 399.

(52) Janssen, R. A. J.; Smilowitz, L.; Sariciftci, N. S.; Moses, D., J. Chem.Phys. 1994, 101, 1787.

(53) Liao, H.-H.; Yang, C.-M.; Wu, C.-H.; Horng, S.-F.; Lee, W.S.; Meng, H.-F.; Shy, J.-T.; Hsu, C.-S., Appl. Phys. Lett. 2007, 90 , 013504 .

(54) Moiseev, A. G.; Margulies, E. A.; Schneider, J. A.; BelangerGariepy, F.; Perepichka, D. F., Dalton Trans. 2014, 43, 2676.

(55) Whited, M. T.; Djurovich, P. I.; Roberts, S. T.; Durrell, A. C.; Schlenker, C. W.; Bradforth, S. E.; Thompson, M. E., J. Am. Chem. Soc. 2011, 133, 88 .

(56) Baldo, M. A.; Forrest, S. R., Phys. Rev. B 2000, 62, 10958.

(57) Monkman, A. P.; Burrows, H. D.; Hartwell, L. J.; Horsburgh,

L. E.; Hamblett, I.; Navaratnam, S., Phys. Rev. Lett. 2001, 86, 1358.

(58) Köhler, A.; Beljonne, D., Adv. Funct. Mater. 2004, 14, 11. 
1

2

3

4

5

6

7

8

9

10

11

12

13

14

15

16

17

18

19

20

21

22

23

24

25

26

27

28

29

30

31

32

33

34

35

36

37

38

39

40

41

42

43

44

45

46

47

48

49

50

51

52

53

54

55

56

57

58

59

60
Insert Table of Contents artwork here

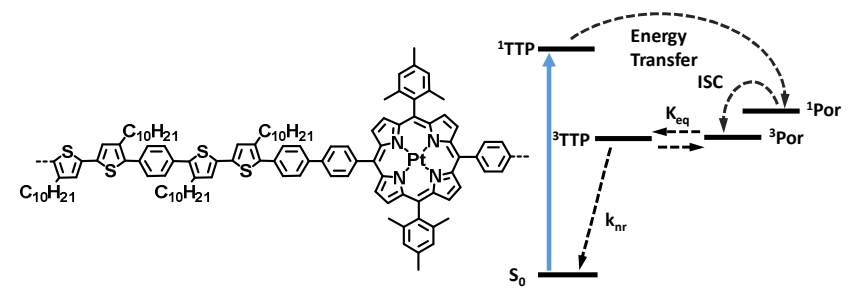

